\title{
A susceptibility gene for premature ovarian failure (POF) maps to proximal Xq28
}

\author{
Francesca Rossetti ${ }^{1}$, Flavio Rizzolio ${ }^{1}$, Tiziano Pramparo ${ }^{2}$, Cinzia Sala ${ }^{1}$, Silvia Bione ${ }^{3}$, \\ Franca Bernardi ${ }^{4}$, Mara Goegan ${ }^{1}$, Orsetta Zuffardi $^{2}$ and Daniela Toniolo*,
}

${ }^{1}$ Dibit-San Raffaele Scientific Institute, Milano, Italy; ${ }^{2}$ Department of Pathology and Medical Genetics, University of Pavia, Pavia, Italy; ${ }^{3}$ Institute of Molecular Genetics-CNR, Pavia, Italy; ${ }^{4}$ Service of Neonatal Pathology and Genetics, Borgo Roma Hospital, Verona, Italy

Terminal deletions of the long arm of the human $X$ chromosome have been described in women with premature ovarian failure (POF). We report here the molecular characterization of an inherited deletion in two affected women and in their mother. The two daughters presented secondary amenorrhea at 17 or 22 years respectively, while the mother was fertile. She had four children, but she eventually had premature menopause at $\mathbf{4 3}$ years of age. The fine molecular analysis of the deletion showed that the three women carried an identical deletion. We conclude that the phenotypic difference within the family must be attributed to genetic or environmental factors and not to the presence of different extent deletions. By comparison with other deletions in the region, we map a susceptibility gene for POF to $4.5 \mathrm{Mb}$, in the distal part of Xq.

European Journal of Human Genetics (2004) 12, 829-834. doi:10.1038/sj.ejhg.5201186

Published online 28 July 2004

Keywords: POF; turner syndrome; Xq deletions

\section{Introduction}

Genes for ovulation have been hypothesized on the $\mathrm{X}$ chromosome. X chromosome monosomy, or Turner syndrome (TS), is the only chromosome monosomy compatible with life and is characterized by short stature, ovarian dysgenesis and a number of anatomical and physiological manifestations. ${ }^{1}$ It was suggested that TS is due to monosomy for genes required in double dose in female subjects. ${ }^{2,3}$ Accordingly, it was shown that short stature is due to monosomy for a gene, SHOX, in the short arm pseudoautosomal region. ${ }^{4}$ The causes of ovarian dysgenesis are not clarified. In TS, the gonads differentiate normally until about the third month of gestation when the oocytes start to accelerate their apoptosis resulting in a decrease in

${ }^{*}$ Correspondence: Dr D Toniolo, Dibit-San Raffaele Scientific Institute, Milano, Italy, Via Olgettina 58, 20132 Milano, Italy.

Tel: + 3902 26434764; Fax: + 3902 26434767;

E-mail: daniela.toniolo@hsr.it

Received 26 November 2003; revised 9 January 2003; accepted 6 February 2004 the final number of oocytes, an increase in ovarian fibrosis and in ovarian failure. As a consequence, in the majority of adult TS female subjects ovaries appear as streaks. Partial monosomies of the long or of the short arm are also associated with ovarian failure: the phenotype is less severe and they are commonly associated with secondary amenorrhea, presence of reduced size ovaries and of a reduced number of follicles. ${ }^{5,6}$ As different regions of the chromosome may be deleted, alterations in gene dosage of several genes along the whole chromosome appear responsible for ovarian failure. Many genes escaping $X$ chromosome inactivation are in $\mathrm{Xp}$, and they may be responsible for ovarian failure due to Xp monosomy. ${ }^{7}$ In $\mathrm{Xq}$, fewer genes escape $\mathrm{X}$ inactivation, but ovarian function may still be impaired by monosomy for genes required in double dose after $\mathrm{X}$ chromosome reactivation for germ-cell development.

Some of the genes for POF may be localized in the 'critical region for premature ovarian failure', spanning from Xq13 to Xq26/27 and defined from partial deletions, 
X-autosome balanced translocations and other chromosome rearrangements, occurring on the long arm of the chromosome and frequently associated with the disorder. ${ }^{5,6}$ However, whether the same genes are involved in $\mathrm{X}$ autosome translocations and in monosomies is an open question, mainly as several independent studies have shown that most balanced translocations do not interrupt genes. $^{8-10}$

The partial $\mathrm{X}$ chromosome monosomies are always associated with preferential inactivation of the deleted chromosome and frequently involve most of the q- or parm and some TS stigmata. Interstitial deletions and terminal deletions only associated with POF have also been described. ${ }^{11-13}$ The smallest deletions were described on the distal long arm of the $\mathrm{X}$ chromosome, with breakpoints in Xq26/27. ${ }^{14-16}$ None of the carriers of this group of deletions displayed TS or any other TS stigmata, but the phenotypes were rather variable. Some of the carriers had numerous children. Some had irregular menstruation and secondary amenorrhea. Few had premature menopause after 40 years of age. One case had primary amenorrhea. In few instances they were found in families, segregating with different temporal onset of ovarian failure. Since they were described many years ago, no detailed molecular characterization is available and we cannot exclude that the phenotypic differences are due to differences in the extent of the deletions and of the number of genes involved.

One family from Italy, family $\mathrm{M}$, was previously reported as $46, \mathrm{X}$, del(X)(q27). ${ }^{16}$ The three women in the family did not have signs of TS. Two affected women carried a terminal deletion of $\mathrm{Xq}$ and presented secondary amenorrhea at 17 or 22 years of age, after a period of oligomenorrhea. The deletion was inherited from the mother who had four children but eventually had premature menopause at 43 years of age. The deleted $X$ was inactivated. We have analyzed in detail the deletion in this family and we now report the molecular characterization leading to suggestion of a susceptibility gene for POF in Xq28.

\section{Materials and methods Patients and DNAs}

The three women carrying the deletion in family $\mathrm{M}$ were described previously (patients 12-14 from Maraschio et al). ${ }^{16}$ They had normal height $(160-168 \mathrm{~cm})$ and none of the TS traits. The mother, I-1, had menarche at 14 years of age. She had periods of amenorrhea alternating with regular menstruation. This allowed her to have four children at 20,25, 27 and 32 years of age. At 43 years of age she entered menopause. The daughter II-1, had menarche at 15 years of age and she had always irregular cycles until 17 years of age when she entered menopause. The second daughter, II-2, had menarche at 13, irregular cycles until the age of 19 years when she started taking the pill. At 22 years, she stopped the pill and she had amenorrhea thereafter. The three women referred to have had high FSH and LH levels at diagnosis, but the data were not available at the time of the study. The last daughter, II4 , had a normal caryotype. She had menarche at 14 years of age and she always had regular cycles. She has two children.

DNA was prepared from blood or lymphoblastoid cell cultures using standard methods. For pulsed field gel electrophoresis (PFGE) analysis, the DNAs were digested with the enzymes indicated in the conditions suggested by the suppliers (Promega) and fractionated in 1\% agarose gels at $180 \mathrm{~V} / 170 \mathrm{~mA}$ for $20 \mathrm{~h}$ and $5 \mathrm{~s}$ pulses.

\section{Heterozygosity mapping}

The extent of the deletion was determined by heterozygosity mapping using microsatellites. Some of the microsatellites analyzed (DXS1227, DXS8043, DXS8091, DXS1073) were from the ABIPrism ${ }^{\mathrm{TM}}$ linkage mapping set. The PCRs were carried out as suggested by the supplier (ABI) and the analysis was performed by capillary electrophoresis, using the MegaBace and analyzed by the MegaBace Genetic Profiler (Amersham Pharmacia Biotech).

Additional microsatellites were selected using the Ensembl browser (http://www.ensembl.org/). Primer sequence, PCR amplification conditions and heterozygosity of the microsatellites used are described in Table 1. Amplification products were analyzed by radioactive labeling the PCR reaction using ${ }^{32} \mathrm{P} \mathrm{dCTP}(0.25 \mu \mathrm{Ci} / 25 \mu \mathrm{l}$ reaction) and fractionation in $7 \%$ denaturing acrylamide gel electrophoresis. Position of the microsatellites and of the genomic clones used in FISH experiments was derived from Ensemble (release of 4-11-2003).

\section{FISH analysis}

BAC and PAC clones were selected from Ensembl (http:// www.ensembl. org/). Cosmid clones were characterized in our laboratory. ${ }^{17,18}$ DNA was extracted from BAC and PAC clones using the PhasePrep BAC DNA kit (SIGMA) and labeled by nick translation (Roche Diagnostic, IN, USA) with biotin-dUTP or dig-dUTP (Roche). FISH experiments were performed by conventional methods. Briefly, the probes for each slide were combined as required (400 ng per slide), dried down and suspended in 50\% formamide, 1\% Tween-20, 20\% dextran sulfate along with salmon sperm DNA and Cot- 1 DNA, then denatured at $70^{\circ} \mathrm{C}$ for $10 \mathrm{~min}$, preannealed at $37^{\circ} \mathrm{C}$ for $15 \mathrm{~min}$, and hybridized with denatured slides at $37^{\circ} \mathrm{C}$. Post-hybridization washes were $50 \%$ formamide at $42^{\circ} \mathrm{C}$ for $15 \mathrm{~min}, 2 \mathrm{xSSC}$ at $37^{\circ} \mathrm{C}$ for $8 \mathrm{~min}$, and $1 \mathrm{xPBD}$ at room temperature for $2 \mathrm{~min}$. The signals were amplified with FICT-avidin (Sigma) and antiavidin (Sigma) for the biotin-dUTP, and with anti-digoxigenin monoclonal antibody, anti-mouse IgG-digoxigenin and anti-digoxigenin-rhodamine FAB fragments (all from Sigma) for dig-dUTP. Slides were counterstained with DAPI 
Table 1 Primers for heterozygosity mapping

\begin{tabular}{|c|c|c|c|}
\hline & Primer sequence & Annealing temp. $\left({ }^{\circ} \mathrm{C}\right)$ & $\mathrm{Het}^{\mathrm{a}}$ \\
\hline DXS6751 & $\begin{array}{l}\text { F: 5'-CAGAGCTCATCACACATAGG-3' } \\
\text { R: 5'-GTGCACATGGCATAAAATCCG-3' }\end{array}$ & 53 & ND \\
\hline AFMB323XG1 & $\begin{array}{l}\text { F: 5'-GAGTTCCTACATATGTACACC-3' } \\
\text { R: 5'-CCCAGATGTGAGCTGTTGAC-3' }\end{array}$ & 55 & ND \\
\hline DXS8106 & $\begin{array}{l}\text { F: 5'-AATTCATCTGCTCCTAATGC-3' } \\
\text { R: 5'-AGCTGTAGAGTTGAGGAATG-3' }\end{array}$ & 57 & 0.6917 \\
\hline DXS8069 & $\begin{array}{l}\text { F: 5'-AACAGTCATTGTAGGCATCG-3' } \\
\text { R: 5'-GAATTGCCAGTCATCCC-3' }\end{array}$ & 58 & 0.62 \\
\hline DXS1684 & $\begin{array}{l}\text { F: 5'-AGCACCCAGTAAGAGACTGAAC-3' } \\
\text { R: 5'-CCTCAGTGGCAACCACTCAAG-3' }\end{array}$ & 57 & 0.8176 \\
\hline DXS8061 & $\begin{array}{l}\text { F: 5'-GCTTGAAGTGTCCATGAGGTATC-3' } \\
\text { R: 5'-AGAAGCTGATGTGCTCCCTG-3' }\end{array}$ & 57 & 0.6903 \\
\hline DXS15 & $\begin{array}{l}\text { F: 5'-AGCACATGGTATAATGAACCTCCACG-3' } \\
\text { R: 5'-CAGTGTGAGTAGCATGCTAGCATTT-3' }\end{array}$ & 65 & 0.84 \\
\hline DXS1108 & $\begin{array}{l}\text { F: 5'-ATTCATCATATGTGATTTCCACAGCC-3' } \\
\text { R: 5'-ACTAGGCGACTAATACAGTGGTGC-3' }\end{array}$ & 65 & 0.75 \\
\hline
\end{tabular}

${ }^{a}$ Het indicates Heterozygote frequency among Caucasians.

(Sigma) and mounted in Vectashield antifading medium (Vector Laboratories, USA). Signals were visualized under a Nikon E1000 microscope equipped with a cooled chargecoupled device (CCD) camera and the Genikon image analysis software.

\section{Results}

\section{Heterozygosity mapping}

The extent of the deletions in the three affected women of family $\mathrm{M}$ was defined by heterozygosity mapping using microsatellite markers spanning Xq26-Xq28 (Figure 1). The DNA of the three affected women, of a normal daughter and of a normal son, were analyzed. The three women carrying the deletion were heterozygotes for marker AFMB323Xg1, but they had only one of the alleles for marker DXS8106 in Xq27.3, at $140.9 \mathrm{Mb}$ and of all the other tested until DXS15 in Xq28, at $151.0 \mathrm{Mb}$. The last two markers tested, DXS1073 and DXS1108 were both present in heterozygote state in the three women. The deletion is therefore interstitial and the last $3 \mathrm{Mb}$ of $\mathrm{Xq} 28$ are present in the three patients.

\section{FISH mapping}

To further define the deletion BAC, PAC and cosmid clones spanning the breakpoint regions were used in FISH analysis (Table 2 and Figure 2). The proximal breakpoint in all the three women was mapped in Xq27.3 between the BACs RP11-485I9, which is present on both chromosomes, and RP11-156M16, which is absent from the deleted chromosome. The two BACs define a breakpoint interval of $200 \mathrm{~kb}$, identical in the three women, that was not defined further as analysis of the region showed that it is gene free. The breakpoint is $900 \mathrm{~kb}$ distal from the MAGEE1 gene and $500 \mathrm{~kb}$ from the gene $N M-173078$ of unknown function, which is deleted.

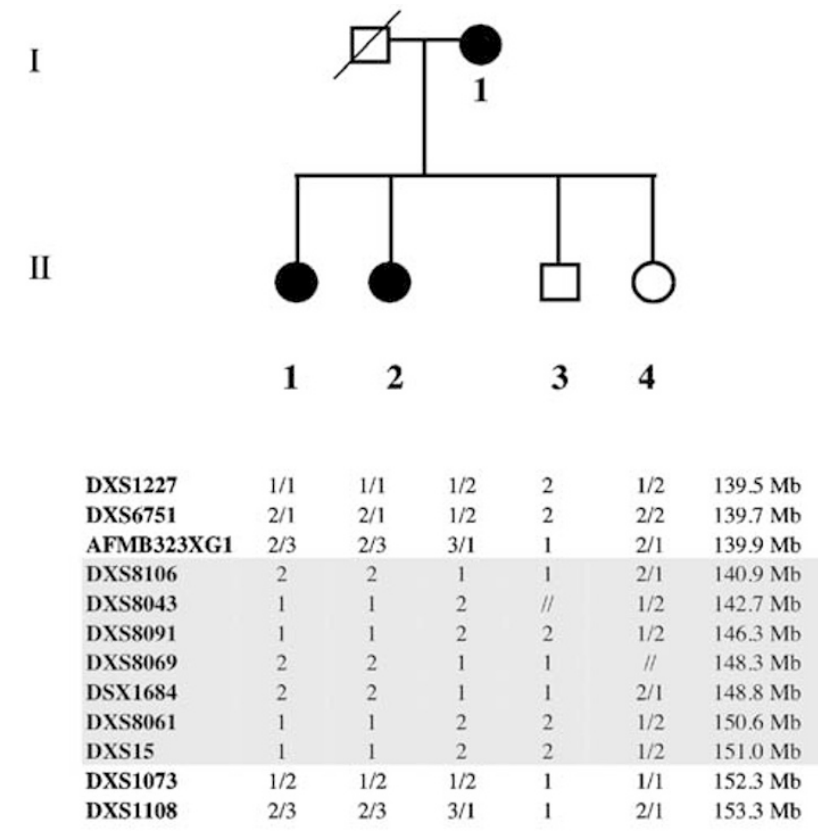

Figure 1 Heterozygosity mapping with microsatellites. Under the family tree are the genotypes of each member of family M. Different alleles have different numbers and the position in $\mathrm{Mb}$ of each microsatellite along the $X$ chromosome sequence is indicated.

In $\mathrm{Xq} 28$, the breakpoint was mapped between cosmid Qc-12B2, that is absent and the overlapping cosmids QcN2215 and Qc-P0113, both present. Accordingly, two-color FISH on the deleted chromosome (Figure 2) shows merging signals with clones RP11-485I9 and Qc-P0113 flanking the deletion (Table 2) and no signals with clones RP5-1185M13 and Qc-L074, both in the deletion (Table 2). The sequence of the deleted cosmid, Qc-12B2, is included in the contig U52112, between 151.58 and $151.76 \mathrm{Mb}$ of the $\mathrm{X}$ chromo- 
Table 2 FISH analysis

\begin{tabular}{lcccc}
\hline Clone name & Position $(\mathrm{Mb})$ & RT & MM & MS1 \\
\hline RP11-73D6 & 140.3 & $+/+$ & $+/+$ & $+/+$ \\
RP11-260C6 & 140.4 & $+/+$ & $+/+$ & $+/+$ \\
RP11-485I9 & 140.5 & $+/+$ & $+/+$ & $+/+$ \\
RP11-156M16 & 140.8 & $+/-$ & $+/-$ & $+/-$ \\
RP5-1185M13 & 140.9 & $+/-$ & $+/-$ & $+/-$ \\
QC-12B2 & 151.7 & $+/-$ & $+/-$ & $+/-$ \\
QC-L074 & 151.8 & $+/-$ & $+/-$ & $+/-$ \\
QC-N2215 & 151.98 & $+/+$ & $+/+$ & $+/+$ \\
QC-P0113 & 152.02 & $+/+$ & $+/+$ & $+/+$ \\
\hline
\end{tabular}

$+/+=$ signal on both chromosomes; $+/-=$ signal on one chromosome.

a

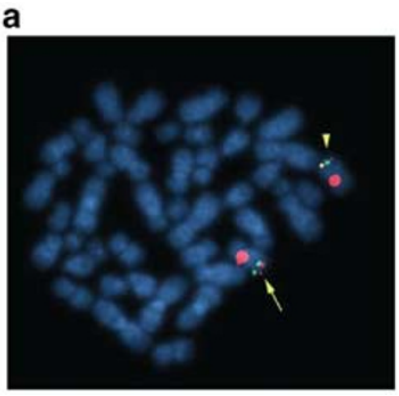

b

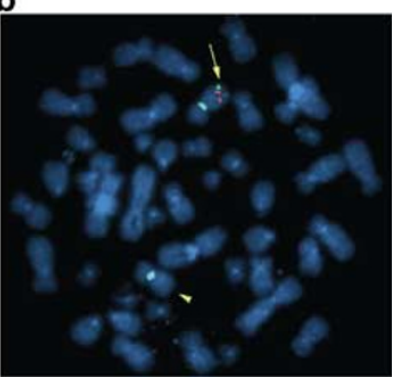

Figure 2 Two-color FISH analysis of the deletion. (a) In situ hybridization with clones RP11-48519 (green) and Qc-PO113 (red) delimiting the deletion. The yellow arrow indicates the normal $X$ chromosome with two distinct signals. The yellow arrowhead shows the deleted chromosome $X$ with merging signals. An alpha- $X$ specific clone was hybridized as a control probe (red). (b) In situ hybridization with clones RP5-1185M13 (red) and Qc-LO74 (green) located within the deleted region. The yellow arrow indicates the normal $X$ chromosome with two distinct signals. The yellow arrowhead shows the deleted chromosome $X$ with no signals. An alpha- $X$ specific clone was hybridized as a control probe (green).

some. The ends of cosmids Qc-N2215 and Qc-P0113 were sequenced. Comparison with the human genomic sequence positioned the cosmids $4 \mathrm{~kb}$ distal to the 3 ' end of the color vision gene cluster. In Xq28 the distal breakpoints was therefore mapped to a $200 \mathrm{~kb}$ region containing the MECP2 gene and the OPN, and the CXorf2 genes cluster (Figure 3a).

PFGE analysis of the Xq28 breakpoint

The breakpoint in Xq28 was further refined by PFGE and Southern blot analysis. From the restriction map of the region, the DNAs of the three patients and of male and female controls were digested with EcoRV and KpnI and hybridized with the probes indicated in Figure 3a. All the probes tested (MIR2-IR6) were deleted in the patients as

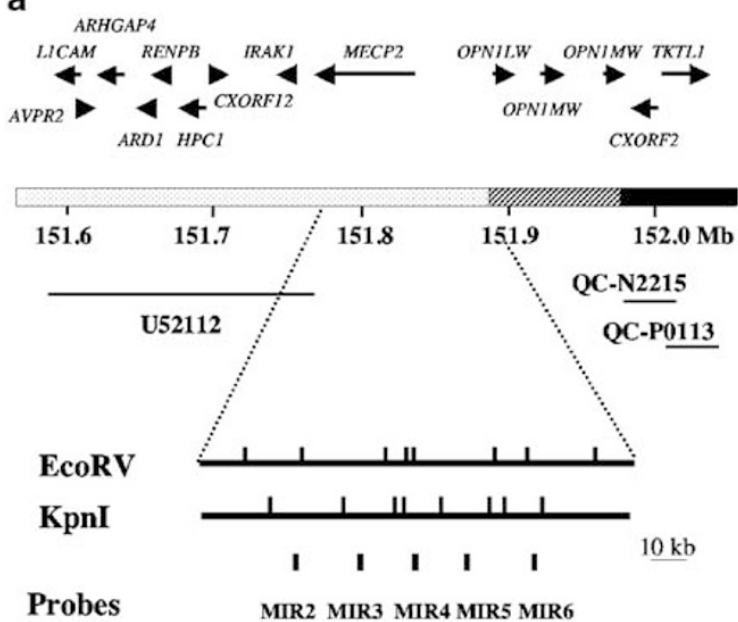

b

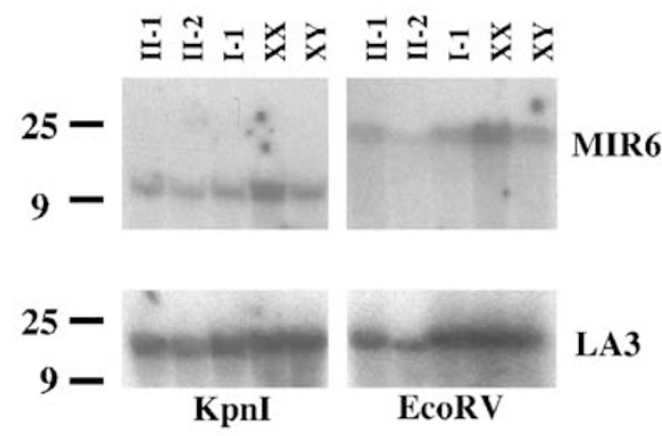

Figure 3 Fine analysis of the Xq28 deletion breakpoint. (a) map of the region: the dotted bar indicates the deleted region, the striped bar is the region of the breakpoint, in black is the nondeleted region. Above are the genes of the region. Below is the position of the restriction enzyme site used in PFGE, and the probes used. (b) Southern blot analysis of the Xq28 breakpoint. Above are the DNA used in the analysis and below are the enzymes used for DNA digestion. MIR6 is the most distal probe used that identified the region flanking the OPN1LW gene. LA3 is a probe from chromosome 18 hybridized to the same filter to control for the amount of DNA in each lane.

shown by the decreased intensity of he band compared to the control DNAs (Figure $3 b$ ). The results show that the distal breakpoint in all three patients is identical and is localized within the OPN/Cxorf2 gene cluster.

\section{Discussion}

We report the fine mapping of an interstitial deletion of the $\mathrm{X}$ chromosome in family $\mathrm{M}^{16}$ The deletion was associated with early onset ovarian failure and sterility in two sisters. A third sister had a normal karyotype. She had regular cycles and two children. The deletion was inherited 
from the mother who had a much milder phenotype as she had four children. The mother had, however, irregular menses and early menopause at the age of 43 years. We show here that the three women carry an identical deletion. Despite their very different phenotype, the results exclude the possibility that recombination occurring in the mother has produced a larger deletion responsible for the earlier onset of POF in the two daughters.

Few other deletions in distal $\mathrm{Xq}$ have been reported that were associated with POF. Two sisters ${ }^{14}$ presented a deletion similar to that in family $\mathrm{M}$, from Xq26/27 to qter: one sister, $\mathrm{KS}$, entered menopause at 21 years of age, the other (MK) had very irregular menstrual periods for many years and was still irregularly menstruating at 40 years of age, the time of the report. She had a normal son. The mother was dead when the family was studied, but she was reported as normal except for early onset of menopause at 25 years of age. A third family was described by Trunca et al: ${ }^{15}$ the patient had irregular menses for few years and amenorrhea at 18 years of age. One sister and the mother had the same deletion. The sister was still menstruating regularly at 29 years of age and the mother had menopause at 35 years of age. Mosaics were tentatively excluded in all cases.

The characteristics of the three families and the similar extent of the three deletions led us to conclude that POF may be due to the deletion of the distal portion of $\mathrm{Xq}$, and we suggests that a susceptibility gene for POF maps to distal $\mathrm{Xq}$. The deletion in family $\mathrm{M}$ is rather large $(>11 \mathrm{Mb})$, but it could be compared to that in a published family identified from a male proband presenting FRAXA mental retardation. ${ }^{19}$ The deletion was present in the mother who had skewed inactivation and was healthy and intellectually normal. It was reported that she had undergone menopause at the normal age of 48 years. The deletion contained the FMR1 gene and should not contain any gene for POF. Its distal breakpoint was mapped between DXS1193 at $147.08 \mathrm{Mb}$ and the gene IDS at $147.27 \mathrm{Mb},<300 \mathrm{~kb}$ apart. The two deletions, in family $\mathrm{M}$ and in Wolff et $a l^{19}$, partly overlap in their proximal portion. By comparison, we can therefore reduce the region of susceptibility to POF to 4.5 MB between the IDS gene and the CV genes. The region is very gene rich as more than 60 genes are mapped there. Studies of additional terminal deletions may reduce the number of genes and analysis of candidate genes in the region may allow identification of a novel POF gene. This may determine whether a POF gene is indeed present or POF is due to other causes such as $\mathrm{X}$ inactivation or alterations in chromosome pairing, as suggested. ${ }^{20}$

The results show that the POF phenotype depends form the deletion and from additional factors. One could be $\mathrm{X}$ chromosome inactivation. It is not known at which time of embryo development inactivation nor skewing of inactivation exactly occur. The distal breakpoint of the deletion in family $\mathrm{M}$ was mapped within the OPN gene cluster, encoding the genes for Color Vision. The MECP2 gene, responsible for RETT syndrome, ${ }^{21}$ is the first of many genes deleted in the patients. Some (LICAM, AVPR2, ABCD1, $D K C 1, I D S$ and others) are known to cause severe disorders (http://www.ncbi.nlm.nih.gov/entrez). Thus, the complete skewing for $\mathrm{X}$ inactivation that is characteristics of this type of deletions and was previously reported also for family $M$ must occur early enough in order to produce a normal phenotype, devoid of any other symptom, as found in family $\mathrm{M}^{16}$ However, small differences in the time of skewing of $\mathrm{X}$ inactivation in germ cells or in granulosa cells may alter the number of ovarian follicles and determine the age of onset of POF.

Other possibilities may explain the different phenotypes as other environmental factors, such as those known to affect ovulation ${ }^{20,22}$ and the inheritance of a modifying genetic factor. No evidence for the latter hypothesis could be obtained from the family history, and further experiments are needed to clarify this point.

\section{Acknowledgements}

We thank the members of Family $M$ for participating in the research. We acknowledge the financial contribution of the EU contract QLG21999-00791, MIUR-FIRB RBNE0189HM_005 and MIUR-Genomica Funzionale CNR02.00157.ST97.

\section{References}

1 Zinn AR, Ross JL: Turner syndrome and haploinsufficiency. Curr Opin Genet Dev 1998; 8: 322-327.

2 Fisher EM et al: Homologous ribosomal protein genes on the human $\mathrm{X}$ and $\mathrm{Y}$ chromosomes: escape from $\mathrm{X}$ inactivation and possible implications for Turner syndrome. Cell 1990; 63: $1205-1218$

3 Disteche CM: Escape from $\mathrm{X}$ inactivation in human and mouse. Trends Genet 1995; 11: 17-22.

4 Rao E et al: Pseudoautosomal deletions encompassing a novel homeobox gene cause growth failure in idiopathic short stature and Turner syndrome. Nat Genet 1997; 16: 54-63.

5 Therman E, Susman B: The similarity of phenotypic effects caused by Xp and Xq deletions in the human female: a hypothesis. Hum Genet 1990; 85: 175-183.

6 Therman E, Laxova R, Susman B: The critical region on the human Xq. Hum Genet 1990; 85: 455-461.

7 Carrel L, Cottle AA, Goglin KC, Willard HF: A first-generation Xinactivation profile of the human X chromosome. Proc Natl Acad Sci USA 1999; 96: 14440-14444.

8 Prueitt RL, Chen H, Barnes RI, Zinn AR: Most X;autosome translocations associated with premature ovarian failure do not interrupt X-linked genes. Cytogenet Genome Res 2002; 97: 32-38.

9 Prueitt RL, Ross JL, Zinn AR: Physical mapping of nine Xq translocation breakpoints and identification of XPNPEP2 as a premature ovarian failure candidate gene. Cytogenet Cell Genet 2000; 89: 44-50.

10 Mumm S et al: X/autosomal translocations in the Xq critical region associated with premature ovarian failure fall within and outside genes. Genomics 2001; 76: 30-36.

11 Davison RM et al: A familial case of X chromosome deletion ascertained by cytogenetic screening of women with premature ovarian failure. Hum Reprod 1998; 13: 3039-3041.

12 Krauss CM et al: Familial premature ovarian failure due to an interstitial deletion of the long arm of the X chromosome. $\mathrm{N} \mathrm{Engl}$ J Med 1987; 317: 125-131. 
13 Marozzi A et al: Molecular definition of Xq common-deleted region in patients affected by premature ovarian failure. Hum Genet 2000; 107: 304-311.

14 Schwartz C, Fitch N, Phelan MC, Richer CL, Stevenson R: Two sisters with a distal deletion at the Xq26/Xq27 interface: DNA studies indicate that the gene locus for factor IX is present. Hum Genet 1987; 76: 54-57.

15 Trunca C, Therman E, Rosenwaks Z: The phenotypic effects of small, distal Xq deletions. Hum Genet 1984; 68: 87-89.

16 Maraschio P et al: An analysis of Xq deletions. Hum Genet 1996; 97: $375-381$.

17 Maestrini E et al: An archipelago of CpG islands in Xq28: identification and fine mapping of 20 new CpG islands of the human X chromosome. Hum Mol Genet 1992; 1: 275-280.
18 Bione $\mathrm{S}$ et al: Transcriptional organization of a $450-\mathrm{kb}$ region of the human X chromosome in Xq28. Proc Natl Acad Sci USA 1993; 90: $10977-10981$.

19 Wolff DJ et al: Title: Deletions in Xq263-q27.3 including FMR1 result in a severe phenotype in a male and variable phenotypes in females depending upon the X inactivation pattern. Hum Genet 1997; 100: 256-261.

20 Bione S, Toniolo D: X chromosome genes and premature ovarian failure. Semin Reprod Med 2000; 18: 51-57.

21 Amir RE et al: Rett syndrome is caused by mutations in X-linked MECP2, encoding methyl-CpG-binding protein 2. Nat Genet 1999; 23: $185-188$

22 Davis CJ, Davison RM, Conway GS: Genetic basis of premature ovarian failure. Hum Fertil (Camb) 1998; 1: 20-22. 